\title{
OPEN Associations between the dietary inflammatory index and urinary incontinence among women younger than 65 years
}

Shiyu Zhang ${ }^{1,4}$, Haiyang Bian ${ }^{2,4}$, Shi Qiu ${ }^{1,3,4}$, Boyu Cai ${ }^{1}$, Kun Jin ${ }^{1}$, Xiaonan Zheng ${ }^{1}$, Jiakun $\mathrm{Li}^{1}$, Xiang $\mathrm{Tu}^{1}{ }^{1}$, Jianzhong $\mathrm{Ai}^{1}$, Lu Yang $^{1 \bowtie}$ \& Qiang Wei ${ }^{1 凶}$

The purpose of this study was to evaluate the association between dietary inflammatory index (DII) and urinary incontinence (UI) among a representative sample of the US women. We performed a cross-sectional analysis of women younger than 65 years using the 1999 to 2016 NHANES (National Health and Nutrition Examination Survey) populations. DII were calculated based on baseline dietary intake using 24-h dietary recalls. Ul was determined and categorized by self-reported questions. Multivariable logistic regression models were used to assess the association between DII and UI. Stratified linear regression models were applied to test for interaction in prespecified subgroup of interest. A total of 13,441 women age between 20 and 65 years were included in the final analysis. Of these participants $3230(24.03 \%)$ complained of urgency UI, $5276(39.25 \%)$ complained of stress UI and 2028 (15.09\%) complained of mixed UI. On multivariate analysis, analysis with DII categorized as quartiles revealed significantly increase odds of urgency $\mathrm{UI}$ in the most pro-inflammatory quartile compared to the most anti-inflammatory quartile (OR 1.24, 95\% Cl 1.07-1.44, P=0.004 for trend) in full adjustment model. Similar results were observed in SUI (OR 1.14, 95\% CI 1.00-1.30, P=0.021 for trend) and MUI (OR 1.20, 95\% CI 1.02-1.43, $\mathrm{P}=0.022$ for trend). More pro-inflammatory diets, as presented by higher DII scores are associated with an increased likelihood of UI in American women younger than 65 years. Further studies are needed to explore the possible physiological mechanism and evaluate the potential therapeutic implications.

Urinary incontinence (UI) is a common complaint that afflicts $17.1 \%$ of women aged 20 years or older according to a cross-sectional evidence ${ }^{1}$ and the prevalence increases with age ${ }^{2}$. It decreases quality of life, as well as increases significant costs, to both individuals and societies. UI commonly presents as either stress UI, urgency UI, or mixed UI.

Diet, as mixture of pro-inflammatory or anti-inflammatory compounds, is an important potential source and modulator of inflammation ${ }^{3}$. The dietary inflammatory index (DII) was developed to estimate an individual's dietary inflammatory potential using scoring algorithm. It has been construct validate against various inflammatory markers, including C-reactive protein (CRP) $)^{4,5}$, tumor necrosis factor alpha ${ }^{6,7}$, interleukin- $6^{7}$. Moreover, the DII has been shown to be associated with a range of other inflammatory-related conditions including diabetes ${ }^{8}$, cancers $^{9}$, cardiovascular disease ${ }^{10}$, telomere length ${ }^{11}$, chronic kidney disease ${ }^{12}$, frailty ${ }^{13}$, and mortality ${ }^{14}$.

However, there is limited evidence regarding the potential association between DII and urinary incontinence. Therefore, we aim to investigate the cross-sectional association between DII and urinary incontinence using the National Health and Nutrition Examination Survey (NHANES).

\footnotetext{
${ }^{1}$ Department of Urology, Institute of Urology and National Clinical Research Center for Geriatrics, West China Hospital of Sichuan University, No. 37, Guoxue Alley, Chengdu, Sichuan 610041, People's Republic of China. ${ }^{2}$ Institute of Reproductive and Child Health and Department of Epidemiology and Biostatistics, Peking University School of Public Health, Beijing, China. ${ }^{3}$ Center of Biomedical Big Data, West China Hospital, Sichuan University, Chengdu, Sichuan, China. ${ }^{4}$ These authors contributed equally: Shiyu Zhang, Haiyang Bian and Shi Qiu. ${ }^{凶}$ email: wycleflue@163.com; weiqiang163163@163.com
} 


\section{Methods}

Study population. Data from individuals participating in the National Health and Nutritional Examination Surveys (NHANES) (http://www.cdc.gov/nchs/nhanes.htm) were used. NHANES data are cross sectional surveys of the nationally representative, non-institutionalized US population that are sampled in two-year cycles using a complex, stratified, multi-stage, probability cluster design. Questionnaire information is obtained from an in-home interview. Participants are then invited for further examination in their mobile examination clinic (MEC) where standardized physical examination and further questioning conducted.

To increase the sample size we combined the nine survey periods from 1999 to 2016 . The study population was restricted to women, age between 20 and 65 years. We excluded the individuals without complete information on UI $(n=4419)$, missing single 24 -h dietary recall data $(n=1398)$ and missing data for covariate $(n=52)$ (see Supplementary Fig. S1 online). The National Centers for Health Statistics ethics review board approved the protocol and all participants provided written informed consent. All methods were performed in accordance with the relevant guidelines and regulations.

Urinary incontinence. Questions on urinary incontinence were only asked of the 20 years old or older in MECs (Mobile Examination Centers). If participants answered yes to this question, "During the past 12 months, have you leaked or lost control of even a small amount of urine with activity like coughing, lifting, or exercise?" categorized as stress UI. Urgency UI was defined based on a positive answer to: "During the past 12 months, have you leaked or lost control of even a small amount of urine with an urge or pressure to urinate and you couldn't get to the toilet fast enough?". Participants were considered mixed UI if they responded yes to both the stress and urgency UI questions.

Dietary inflammation index. Dietary information were obtained from 24-h dietary recall interviews (24HRs), and then processed by using the USDA's Food and Nutrient Database for Dietary Studies (FNDDS) to obtain micro and macronutrients. The $24 \mathrm{HR}$-derived dietary data were used to calculate DII scores for all participants. The DII food parameters available in NHANES database included fat; protein; carbohydrates; vitamins A, B1, B2, B6, B12, C, D, E; niacin; grams of alcohol; omega3 and omega6 polyunsaturated fatty acids; fiber; cholesterol; saturated, monounsaturated, and polyunsaturated fatty acids; folic acid; Fe; Mg; Zn; Se; $\beta$-carotene and caffeine. Higher DII scores indicate more pro-inflammatory diets and more negative values are more antiinflammatory. The development and validation of the DII have been discussed in detail elsewhere ${ }^{15}$.

Covariates. The other possible covariates used for adjustment including age, self-reported race/ethnicity, which was then categorized as non-Hispanic white, non-Hispanic black, Hispanic (including Mexican-American) and other ethnicity. Wealth was assessed using the ratio of family income to poverty [i.e., Poverty Income Ratio (PIR)] which was calculated based on the poverty guidelines of the Department of Health and Human Services. Education was categorized as less than high school, high school/equivalent, or greater than high school. Body mass index was calculated as $\mathrm{kg} / \mathrm{m}^{2}$ and as less than 25.0 (underweight/normal weight), 25.0 to 29.9 (overweight), and 30.0 or more (obese). We used composite cardiovascular disease risk score which aggregating several risk factors including, hypertension, coronary artery disease and/or history of transient ischemic attack or stroke and diabetes. The score ranges from 0 to 5. Participants were categorized as menopausal if they reported no menstrual periods because of menopause. Parity was defined as the number of total cesarean and vaginal deliveries. Other health related variables included cigarette consumption (whether smoked at least 100 cigarettes in lifetime), alcohol consumption and physical activity. All covariates were chosen based on known or suspected confounders of the relationship between DII and urinary incontinence in women.

Statistical analysis. Data are presented as mean \pm SD or proportions. Kruscal Whallis $\mathrm{H}$ test (continuous variables) and chi-square tests (categorical variables) were used to determine if there were any statistical differences among different DII groups (quartiles). We used multivariate linear regression model to evaluate the associations between DII and UI. According to the recommendation of STROBE statement ${ }^{16}$, we showed unadjusted, minimally adjusted and fully adjusted results simultaneously. Generalized additive model (GAM) was applied to adjust for age (smooth), energy (smooth) and deliveries (smooth) in all models to account for potential nonlinearity in the association between these continuous variables and UI. The subgroup analyses were performed using stratified linear regression models. The modification and interaction of subgroup were inspected by the likelihood ration test. All analyses were performed using the statistical software packages R (http://www.R-proje ct.org, The R Foundation) and EmpowerStats (http://www.empowerstats.com, X\&Y Solutions, Inc., Boston, MA). P-values were two sided with a significance level of $<0.05$.

\section{Results}

Baseline characteristics. A total of 13,441 women age between 20 and 65 years were included in the final analysis. Of these women 3230 (24.03\%) complained of UUI, 5276 (39.25\%) complained of SUI and 2028 (15.09\%) complained of MUI. The DII score in this study ranged from a maximally pro-inflammatory score of +5.33 to maximally anti-inflammatory score of -4.81 . Supplementary Table S1 online displays population characteristics by DII quartiles. Those in the DII quartile 4 were more likely to be younger, non-Hispanic Black, and have lower energy intakes, lower alcohol intake per week, lower family income, lower educational attainment, higher BMI, and higher proportion of living alone, less physical activity, higher proportion of menopause, more cigarettes, more deliveries, and were more risky to CVD compared to those in DII quartile 1. 


\begin{tabular}{|c|c|c|c|c|c|c|}
\hline & \multicolumn{4}{|l|}{ Quartile of DII } & \multirow[b]{3}{*}{$P$ value for trend } & \multirow[b]{3}{*}{ DII (continuous) } \\
\hline & 1 & 2 & 3 & 4 & & \\
\hline & -4.81 to 0.61 & $0.61-2.11$ & 2.11-3.27 & $3.27-5.33$ & & \\
\hline \multicolumn{7}{|c|}{ UUI (OR, 95\%CI, P) } \\
\hline Model1 & Ref & $\begin{array}{l}1.06(0.95,1.18) \\
0.328\end{array}$ & $1.08(0.96,1.21) 0.187$ & $\begin{array}{l}1.11(1.00,1.25) \\
0.06\end{array}$ & 0.055 & $\begin{array}{l}1.02(1.00,1.04) \\
0.094\end{array}$ \\
\hline Model2 & Ref & $\begin{array}{l}1.17(1.04,1.32) \\
0.010\end{array}$ & $\begin{array}{l}1.31(1.15,1.48) \\
<0.001\end{array}$ & $\begin{array}{l}1.53(1.33,1.76) \\
<0.001\end{array}$ & $<0.001$ & $\begin{array}{l}1.08(1.06,1.11) \\
<0.001\end{array}$ \\
\hline Model3 & Ref & $\begin{array}{l}1.07(0.94,1.20) \\
0.308\end{array}$ & $\begin{array}{l}1.13(0.99,1.28) \\
0.066\end{array}$ & $\begin{array}{l}1.24(1.07,1.44) \\
0.003\end{array}$ & 0.004 & $\begin{array}{l}1.04(1.01,1.06) \\
0.017\end{array}$ \\
\hline \multicolumn{7}{|c|}{ SUI (OR, 95\%CI, P) } \\
\hline Model1 & Ref & $\begin{array}{l}0.98(0.89,1.08) \\
0.62\end{array}$ & $\begin{array}{l}0.94(0.85,1.04) \\
0.232\end{array}$ & $\begin{array}{l}0.82(0.74,0.91) \\
<0.001\end{array}$ & 0.002 & $\begin{array}{l}0.97(0.95,0.99) \\
0.001\end{array}$ \\
\hline Model2 & Ref & $\begin{array}{l}1.15(1.04,1.28) \\
0.009\end{array}$ & $\begin{array}{l}1.26(1.13,1.41) \\
<0.001\end{array}$ & $\begin{array}{l}1.30(1.14,1.47) \\
<0.001\end{array}$ & $<0.001$ & $\begin{array}{l}1.07(1.04,1.09) \\
<0.001\end{array}$ \\
\hline Model3 & Ref & $\begin{array}{l}1.08(0.97,1.21) \\
0.138\end{array}$ & $\begin{array}{l}1.15(1.02,1.28) \\
0.019\end{array}$ & $\begin{array}{l}1.14(1.00,1.30) \\
0.05\end{array}$ & 0.021 & $\begin{array}{l}1.03(1.01,1.06) \\
0.011\end{array}$ \\
\hline \multicolumn{7}{|c|}{ MUI (OR, 95\%CI, P) } \\
\hline Model1 & Ref & $\begin{array}{l}1.02(0.90,1.17) \\
0.732\end{array}$ & $\begin{array}{l}1.05(0.92,1.20) \\
0.474\end{array}$ & $\begin{array}{l}1.03(0.90,1.18) \\
0.660\end{array}$ & 0.565 & $\begin{array}{l}1.01(0.98,1.03) \\
0.540\end{array}$ \\
\hline Model2 & Ref & $\begin{array}{l}1.20(1.05,1.39) \\
0.01\end{array}$ & $\begin{array}{l}1.39(1.20,1.61) \\
<0.001\end{array}$ & $\begin{array}{l}1.57(1.34,1.85) \\
<0.001\end{array}$ & $<0.001$ & $\begin{array}{l}1.10(1.07,1.14) \\
<0.001\end{array}$ \\
\hline Model3 & Ref & $\begin{array}{l}1.08(0.93,1.24) \\
0.318\end{array}$ & $\begin{array}{l}1.16(1.00,1.35) \\
0.056\end{array}$ & $\begin{array}{l}1.20(1.02,1.43) \\
0.033\end{array}$ & 0.022 & $\begin{array}{l}1.04(1.00,1.07) \\
0.034\end{array}$ \\
\hline
\end{tabular}

Table 1. Relationship between DII and urinary incontinence in the unadjusted, minimally adjusted and fully adjusted logistic regression models. Model1: adjust for: None. Model2: adjust for: age (Smooth); Race; Energy (Smooth). Model3: adjust for: age (Smooth); Race; Energy (Smooth); BMI; menopause; alcohol intake per week; CAD score; physical activity; least 100 cigarettes; Ratio of family income to poverty; education; Marital; deliveries (Smooth). DII, dietary inflammatory index; UUI, Urge Urinary Incontinence; SUI, stress urinary incontinence; MUI, mixed urinary incontinence; CI, confidence interval; Ref, Reference; OR, odds ratio.

Associations of dietary inflammation index with urinary incontinence. ORs for UI by quartile categories and one-unit increment in DII score are shown in Table 1. On multivariate analysis, when analyzing DII as a continuous variable, DII was not statistically significantly associated with UUI in crude model (OR $1.02,95 \%$ CI 1.00-1.04, $\mathrm{P}=0.094)$. However, after adjusting for age, race, energy, a one-unit increase in DII (more pro-inflammatory) was associated with significantly greater odds of UUI (OR 1.08, 95\% CI 1.06-1.11, $\mathrm{P}<0.001$ ). After full adjustment for all covariates, the OR was slightly attenuated (OR 1.04, 95\% CI 1.01-1.06, $\mathrm{P}=0.017)$. Similar results were observed in SUI (OR 1.03, 95\% CI 1.01-1.06, $\mathrm{P}=0.011)$ and MUI (OR 1.04, $95 \%$ CI 1.00-1.07, $\mathrm{P}=0.034)$. Analysis with DII categorized as quartiles revealed significantly increase odds of UUI in quartile 4 (most pro-inflammatory) compared to quartile 1 (most anti-inflammatory) (OR $1.24,95 \%$ CI $1.07-1.44, \mathrm{P}=0.004$ for trend) in full adjustment model. In SUI and MUI, significantly associations with DII were observed when comparing participants in the highest versus the lowest DII group quartile. (OR 1.14, 95\% CI $1.00-1.30, \mathrm{P}=0.021$ for trend; $\mathrm{OR} 1.20,95 \% \mathrm{CI} 1.02,-1.43, \mathrm{P}=0.022$ for trend, respectively).

Subgroup analysis. In stratified analyses for UUI with a one-unit increment in DII score, test for interactions were significant for alcohol consumption. ( $P$ for interaction $=0.012$ ), while the test for interactions were not statistically significant for age, race, energy, BMI, menopause, CAD score, physical activity, least 100 cigarettes, PIR, education, marital status, deliveries (see Supplementary Table S2 online). In stratified analyses for SUI, stronger positive associations were found in those who have vigorous physical activity, at least 100 cigarettes consumption and in postmenopausal women ( $P$ for interaction $=0.046,0.003$ and 0.027 respectively) (see Supplementary Table S3 online). No significant interactions were seen for other covariates on the associations between SUI and DII. Alcohol and cigarettes consumption, education attainment seems to be effect modification factors on the association between MUI and DII ( $\mathrm{P}$ for interaction $=0.022,0.011$ and 0.035 respectively) (see Supplementary Table S4 online).

\section{Discussion}

In this cross-sectional study using a nationally representative sample of women in the United States the women age between 20 and 65 years with more pro-inflammatory diets, as indicated by higher DII scores, are associated with an increased likelihood of UUI, SUI and MUI after adjusting for demographic and health-related covariates.

Several researches have focused on the associations between inflammatory and overactive bladder (OAB). UUI is a common symptom of overactive bladder $(\mathrm{OAB})$ syndrome. Previous studies indicated a prevalence of $82.9 \%$ of UUI in OAB patients ${ }^{17}$. One study examined a large population among men and women aged 30-79 years from the city of Boston. Results showed a consistent association of increasing CRP levels and OAB among both men and women ${ }^{18}$. Results from the Boston Area Community Health study also showed a significant 
association of CRP levels with LUTS (including UUI and OAB) in women ${ }^{19}$. Several small clinical studies have also revealed a potential role of inflammation in $\mathrm{OAB}$ in women ${ }^{20,21}$. Unfortunately, very limited studies have explored the association between inflammatory potential and SUI or MUI in women directly. One study compared the serum proteomic profile in patients with SUI $(n=19)$ with healthy controls $(n=19)$, results showed that 33 proteins were induced in SUI sample which involved in inflammatory response, response to coagulation, cellular stress and cytoskeleton stability/motility ${ }^{22}$.

There are many possible explanations for the increased odds of UI among participants with higher DII scores. As we mentioned before, high DII score (representing pro-inflammatory diets) has been positively associated with increased levels of inflammatory markers including CRP, TNF- $\alpha$, IL-6. Studies indicated that inflammatory cytokines play a key role in the modulation of connexins expression and the pathogenesis of urinary bladder dysfunction ${ }^{23,24}$ and inflammatory cytokines were involved in an interaction of overactive parasympathetic and peptidergic/sensory innervations of the bladder with local immune cells ${ }^{21}$. Furthermore, several molecular pathways indicated that inflammation accompanies the changes of SUI in rat model system. SMAD2 is a known downstream mediator of TGF- $\beta$, which plays an important role in tissue inflammation and other disorders, SMAD2 was found upregulated in SUI compared with healthy controls in previous study ${ }^{25}$.

To our knowledge, our study is first to use a food-based DII score which is considered associated with inflammatory potential to associate with likelihood of UI based on a nationally representative sample. We found that more pro-inflammatory diets are associated with a greater likelihood of any type of UI while controlling for key confounders. As lifestyle and behavioral treatment is considered first-line treatment for the most common types of $\mathrm{UI}^{26}$, our findings could be a potential therapeutic implications regarding to lifestyle such as diet. Our study has several limitations. Notably, causality could not be determined as our study is cross-sectional. Secondly, several variables of our study were self-reported and collected in questionnaire format, raising concerns for recall bias and misclassification bias, since there is possibility for respondents cannot accurately discriminate between UUI and SUI. Thirdly, although a previous study reported accurate responses to incontinence questions nearly identical to those in the NHANES questionnaire; the questionnaires using in the NHANES to identify different UI types have not validated yet; hence we need future studies to validate these questionnaires and confirm our results. Additionally, whether severity of any UI types is associated with DII scores is needed to be identified in the future studies.

\section{Conclusions}

Our study shows that more pro-inflammatory diets, as presented by higher DII scores is associated with an increased likelihood of UI in American women younger than 65 years, suggesting inflammation as a potential mechanism linking dietary patterns and UI development. Future studies, especially those with prospective designs, are needed to strengthen our findings, explore the possible pathophysiological mechanism which could provide us more comprehensive understanding of onset and progression of UI and evaluate the potential therapeutic implications.

\section{Data availability}

Data described in the manuscript, code book, and analytic code will be made publicly and freely available without restriction at http://www.cdc.gov/nchs/nhanes.htm.

Received: 1 December 2020; Accepted: 12 April 2021

Published online: 29 April 2021

\section{References}

1. Wu, J. M. et al. Prevalence and trends of symptomatic pelvic floor disorders in U.S. women. Obstet. Gynecol. 123, 141-148. https:// doi.org/10.1097/aog.0000000000000057 (2014).

2. Gyhagen, M., Akervall, S., Molin, M. \& Milsom, I. The effect of childbirth on urinary incontinence: A matched cohort study in women aged 40-64 years. Am. J. Obstet. Gynecol. 221, 322.e321-322.e317. https://doi.org/10.1016/j.ajog.2019.05.022 (2019).

3. Park, Y. M. et al. Obesity mediates the association between Mediterranean diet consumption and insulin resistance and inflammation in US adults. J. Nutr. 147, 563-571. https://doi.org/10.3945/jn.116.243543 (2017).

4. Shivappa, N. et al. A population-based dietary inflammatory index predicts levels of C-reactive protein in the Seasonal Variation of Blood Cholesterol Study (SEASONS). Public Health Nutr. 17, 1825-1833. https://doi.org/10.1017/s1368980013002565 (2014).

5. Julia, C. et al. Long-term associations between inflammatory dietary scores in relation to long-term C-reactive protein status measured 12 years later: Findings from the Supplémentation en Vitamines et Minéraux Antioxydants (SU.VI.MAX) cohort. Br. J. Nutr. 117, 306-314. https://doi.org/10.1017/s0007114517000034 (2017).

6. Tabung, F. K. et al. Construct validation of the dietary inflammatory index among postmenopausal women. Ann. Epidemiol. 25, 398-405. https://doi.org/10.1016/j.annepidem.2015.03.009 (2015).

7. Piccand, E., Vollenweider, P., Guessous, I. \& Marques-Vidal, P. Association between dietary intake and inflammatory markers: Results from the CoLaus study. Public Health Nutr. 22, 498-505. https://doi.org/10.1017/s1368980018002355 (2019).

8. Denova-Gutiérrez, E. et al. Dietary inflammatory index and type 2 diabetes mellitus in adults: The diabetes mellitus survey of Mexico City. Nutrients https://doi.org/10.3390/nu10040385 (2018).

9. Me, F. \& Tf, A. Meta-analysis of the association between dietary inflammatory index (DII) and cancer outcomes. Int. J. Cancer 141, 2215-2227. https://doi.org/10.1002/ijc.30922 (2017).

10. Tyrovolas, S. et al. Dietary inflammatory potential is linked to cardiovascular disease risk burden in the US adult population. Int. J. Cardiol. 240, 409-413. https://doi.org/10.1016/j.ijcard.2017.04.104 (2017).

11. Shivappa, N., Wirth, M. D., Hurley, T. G. \& Hébert, J. R. Association between the dietary inflammatory index (DII) and telomere length and C-reactive protein from the National Health and Nutrition Examination Survey-1999-2002. Mol. Nutr. Food Res. https:// doi.org/10.1002/mnfr.201600630 (2017).

12. Frith, E. et al. Dietary inflammatory index and memory function: Population-based national sample of elderly Americans. Br. J. Nutr. 119, 552-558. https://doi.org/10.1017/s0007114517003804 (2018). 
13. Resciniti, N. V., Lohman, M. C., Wirth, M. D., Shivappa, N. \& Hebert, J. R. Dietary inflammatory index, pre-frailty and frailty among older US adults: Evidence from the national health and nutrition examination survey, 2007-2014. J. Nutr. Health Aging 23(4), 323-329 (2019).

14. Garcia-Arellano, A. et al. Dietary inflammatory index and all-cause mortality in large cohorts: The SUN and PREDIMED studies. Clin. Nutr. 38, 1221-1231. https://doi.org/10.1016/j.clnu.2018.05.003 (2019).

15. Shivappa, N., Steck, S. E., Hurley, T. G., Hussey, J. R. \& Hébert, J. R. Designing and developing a literature-derived, populationbased dietary inflammatory index. Public Health Nutr. 17, 1689-1696. https://doi.org/10.1017/s1368980013002115 (2013).

16. Von Elm, E. et al. The Strengthening the Reporting of Observational Studies in Epidemiology (STROBE) statement: Guidelines for reporting observational studies. Ann. Intern. Med. 147, 573-577. https://doi.org/10.7326/0003-4819-147-8-200710160-00010 (2007).

17. Ww, C., Nh, K., Kk, C., Mh, B. \& Mt, V. Prevalence, evaluation and management of overactive bladder in primary care. BMC Fam. Pract. 10, 8. https://doi.org/10.1186/1471-2296-10-8 (2009).

18. Kupelian, V. et al. Association of overactive bladder and C-reactive protein levels. Results from the Boston Area Community Health (BACH) Survey. BJU Int. 110, 401-407. https://doi.org/10.1111/j.1464-410X.2011.10769.x (2012).

19. Kupelian, V. et al. Association of C-reactive protein and lower urinary tract symptoms in men and women: Results from Boston Area Community Health survey. Urology 73, 950-957. https://doi.org/10.1016/j.urology.2008.12.012 (2009).

20. Compérat, E. et al. Histologic features in the urinary bladder wall affected from neurogenic overactivity-a comparison of inflammation, oedema and fibrosis with and without injection of botulinum toxin type A. Eur. Urol. 50, 1058-1064. https://doi.org/10. 1016/j.eururo.2006.01.025 (2006).

21. Tyagi, P. et al. Urine cytokines suggest an inflammatory response in the overactive bladder: A pilot study. Int. Urol. Nephrol. 42, 629-635. https://doi.org/10.1007/s11255-009-9647-5 (2010).

22. Koch, M. et al. Serum proteomic pattern in female stress urinary incontinence. Electrophoresis 39, 1071-1078. https://doi.org/10. 1002/elps.201700423 (2018).

23. Wang, Z. et al. Inhibition of TNF-a improves the bladder dysfunction that is associated with type 2 diabetes. Diabetes 61, 21342145. https://doi.org/10.2337/db11-1763 (2012).

24. Heinrich, M., Oberbach, A., Schlichting, N., Stolzenburg, J. U. \& Neuhaus, J. Cytokine effects on gap junction communication and connexin expression in human bladder smooth muscle cells and suburothelial myofibroblasts. PLoS ONE 6, e20792. https://doi. org/10.1371/journal.pone.0020792 (2011).

25. Lin, G. et al. Molecular mechanisms related to parturition-induced stress urinary incontinence. Eur. Urol. 55, 1213-1222. https:// doi.org/10.1016/j.eururo.2008.02.027 (2009).

26. Mayor, S. Behavioural therapy is often more effective than drugs for urinary incontinence in women, finds review. BMJ $364,11223$. https://doi.org/10.1136/bmj.11223 (2019).

\section{Acknowledgements}

This work was supported by the National key research and development program of China (Grant No. 2017YFC0908003), National Natural Science Foundation of China (Grant No.81902578, 81974098), China Postdoctoral Science Foundation (2017M612971), Post-doctoral Science Research Foundation of Sichuan University (2020SCU12041), Post-Doctor Research Project, West China Hospital, Sichuan University (2018HXBH085), National Clinical Research Center for Geriatrics, West China Hospital, Sichuan University (Z2018C01) and XingLin Chen (EmpowerStats X\&Y Solutions, Inc, Boston, MA) for providing statistical methodology consultation.

\section{Author contributions}

Protocol/project development: Q.W., L.Y., S.Q. Data collection or management: B.C., K.J., X.Z., J.L., X.T., J.A. Data analysis: S.Q., H.B. Manuscript writing/editing: S.Z. All authors reviewed the manuscript.

\section{Competing interests}

The authors declare no competing interests.

\section{Additional information}

Supplementary Information The online version contains supplementary material available at https://doi.org/ 10.1038/s41598-021-88833-0.

Correspondence and requests for materials should be addressed to L.Y. or Q.W.

Reprints and permissions information is available at www.nature.com/reprints.

Publisher's note Springer Nature remains neutral with regard to jurisdictional claims in published maps and institutional affiliations.

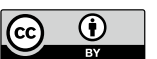

Open Access This article is licensed under a Creative Commons Attribution 4.0 International License, which permits use, sharing, adaptation, distribution and reproduction in any medium or format, as long as you give appropriate credit to the original author(s) and the source, provide a link to the Creative Commons licence, and indicate if changes were made. The images or other third party material in this article are included in the article's Creative Commons licence, unless indicated otherwise in a credit line to the material. If material is not included in the article's Creative Commons licence and your intended use is not permitted by statutory regulation or exceeds the permitted use, you will need to obtain permission directly from the copyright holder. To view a copy of this licence, visit http://creativecommons.org/licenses/by/4.0/.

(c) The Author(s) 2021 